positive ANA (1/640), negative for anti-DNA and anti-ENA antibodies. After etanercept stoppage, skin manifestations resolved but synovitis reappeared. The patient has not been rechallenged. Conclusion Imputability of etanercept in the eruption and biological manifestations described is very likely, given the temporal close correlation between the onset of clinical and biological signs with the initiation of injections, and the resolution following withdrawal of the drug. Further evaluation is needed to know whether if positive ANA or association with other SLE drug inducer are risk factors for development of these TNF inhibitors-induced manifestations.

\section{FRI0094 THE APPLICABILITY OF THE AMERICAN COLLEGE OF RHEUMATOLOGY NOMENCLATURE AND CASE DEFINITION FOR NEUROPSYCHIATRIC LUPUS SYNDROMES. ANALYSIS OF 527 PATIENTS}

LT Costallat, MB Bertolo, S Appenzeller. Internal Medicine-Rheumatology Unit, State University of Campinas, Campinas, Brazil

\subsection{6/annrheumdis-2001.129}

Background The American College of Rheumatology Nomenclature for neuropsychiatric lupus provides case definitions for 19 neuropsychiatric syndromes seen in SLE with reporting standards and recommendations for laboratory and imaging tests.

Objectives To test the new ACR case definitions in a cohort of 527 SLE previously analysed for neuropsychiatric lupus.

Methods Only patients with definite SLE (ACR $>=4$ ) were included. The 19 case definitions established by ACR including diagnostic criteria, important exclusions and methods of ascertainment were utilised for the cohort. Clinical, serologic and imaging data were retrospectively reviewed. The secondary neuropsychiatric manifestations were excluded. A short neuropsychological test battery for the diagnosis of cognitive deficits, and specific tests and questionnaire for headache, mood and anxiety disorders were realised in 40 patients from the cohort and compared to normals. Patients under 14 or older than 65 were not included in this evaluation.

Results Seizures was observed in 39 patients, psychosis in 28, polyneuropathy in 20 , acute confusional state in 15 , cerebrovascular disease in 13 , cranial neuropathy in 8 , mononeuropathy in 7 , myelopathy in 6 , chorea in 4 , asseptic meningitis in 2, Guillan- Barré, demyelinating syndrome and myasthenia gravis in one case each. Autonomic disorders and plexopathy were not observed.

Specific tests applied in 40 patients revealed $75 \%$ of cognitive disturbs, $75 \%$ of mood disorders and $70 \%$ of anxiety disorders. Headache occurred in $62.5 \%$.of these patients.

Conclusion The ACR nomenclature and case definitions for neuropsychiatric lupus is useful for facilitate clinical research, in special multicentric studies and series with a great number of patients. Headache was a common symptom in SLE, however some conditions such as plexopathy or autonomic disorders were rare. Specific battery tests and questionnaire are necessary for cognitive disturbs, mood and anxiety disorders diagnostic but they are not practical for routine testing because are time consuming.

\section{REFERENCE}

1 ACR AD HOC Comitte on neuropsychiatric lupus nomenclature. The American College of Rheumatology nomenclature and case definition for neuropsychiatric syndromes. Arthritis Rheum. 1999;42(4):599-608
FRI0095 COGNITIVE IMPAIRMENT RELATED TO SYSTEMIC LUPUS ERYTHEMATOSUS

S Appenzeller, LT Costallat. Internal Medicine-Rheumatology Unit, State University of Campinas, Campinas, Brazil

10.1136/annrheumdis-2001.130

Background Systemic lupus erythematosus (SLE) is a disease with a broad spectrum of clinical manifestations and a typical course of remission and exacerbation.

Various neuropsychiatric manifestations have been considered to be important features of SLE and indicative of Central Nervous System (CNS) involvement, including cognitive impairment, anxiety and depression.

Objectives To determine the point prevalence of cognitive impairment in patients with SLE. To investigate associations between cognitive impairment and psychiatric disorders, corticosteroid therapy, systemic disease activity and central nervous system (CNS) involvement.

Methods A cross-sectional study of 40 patients with SLE was analysed. To assess cognitive impairment, anxiety and depression standardised psychiatric and clinical research methods were used (Minimental, logic memory tests, short and long memory tests, verbal fluency test, attention tests, BPRS, HAD CAGE and BECK's inventory for measuring depression). Systemic disease activity was measured by SLEDAI. Statistics were performed by chi-square test and by Fisher's exact test.

Results Cognitive impairment was observed in $75 \%$ in SLE. There was no significant relation to the duration of the illness, use of corticotherapy or systemic disease activity. There was a significant association between cognitive impairment and anxiety $(\mathrm{p}<0.05)$ in SLE patients. The same was not observed with depression. CNS involvement was observed in 70\% in SLE. Anxiety was observed in $40 \%$ of CNS-SLE $(p<0.05)$. Cognitive impairment was present in $53.6 \%$ of CNS-SLE and in $66.6 \%$ of non CNS-SLE.

Conclusion The high prevalence of cognitive disorders in our study group could be related to the high prevalence of anxiety, rather than direct involvement of the CNS by SLE. Marked cognitive impairment was present in a significant percentage of patients without CNS involvement.

Supported by FAPESP (Fundação de Amparo à Pesquisa do Estado de São Paulo).

\section{REFERENCES}

1 Bluestein HG. The central nervous system in lupus erythematosus. In: Lahita RG, ed. Systemic lupus erythematosus. New york: Crurchill Livingstone, 1992: 639-55

2 West SG. Neuropsychiatric lupus. Rheum Dis Clin North Am. 1994;20:129-58

3 Bruyin GAW. Controversies in lupus: nervous system involvement. Ann Rheum Dis. 1995;54:159-67

4 Calabrese LV, Stern TA. Neuropsychiatric manifestations of systemic lupus erythematosus. Psychossomatics 1995;36:344-59

5 Wallace DJ, Metzger AL. Systemic lupus erythematosus and the nervous system. In: Wallace DJ, Hanh BH, eds. Dubois lupus erythematosus. Philadelphia: Lea \& Febiger, 1993: 370-85

6 Denburg SD, Carbotte RM, Denburg JÁ. Psychological aspects of systemic lupus erythematosus: cognitive function, mood and self-report. I Rheumatol. 1997;24:998-1003 